\title{
La formación profesional dual en España. Consideraciones sobre los centros que la implementan
}

\author{
Fernando Marbuenda \\ Universitat de Valencia \\ Fernando.Marhuenda@uv.es \\ María José Chisvert \\ Universitat de Valencia \\ Maria.Jose.Chisvert@uv.es \\ Davinia Palomares-Montero \\ Universitat de Valencia \\ Davinia.Palomares@uv.es
}

Resumen: El objetivo de este artículo es analizar el desarrollo de la formación profesional dual en España desde la perspectiva de los profesionales en los centros de formación profesional. A través de dos cuestionarios se recopilará información de la dirección de los centros y de los docentes responsables de implementar esta formación profesional. La muestra abarca todas las regiones y cubre gran parte de las áreas ocupacionales en las que se ha puesto en marcha. Los resultados muestran valoraciones negativas de los participantes y las diferencias entre regiones, sectores profesionales y tipos de centro. Palabras clave: formación profesional dual; alternancia; formación; reforma educativa. 
Dual Vocational Education and Training in Spain. Considering the role of schools involved in the scheme

Abstract: The aim of this paper is to analyze the implementation of dual Vocational Education and Training in Spain from the views of teachers in charge of it in VET Schools. We have used two questionnaires to gather information from both principals as well as teachers responsible for dual schemes. Our sample takes into account all regions in the country, it covers most occupational fields in which dual VET has been developed. Our results show negative assessment of the scheme among participating teachers, varying across regions, professional sectors and types of VET schools.

Keywords: dual Vocational Education; alternance; training; educational reform. 


\section{Planteamiento del problema}

La Ley General de Educación (LGE), aprobada en 1970, asentó una formación profesional (FP en adelante) con cierto carácter de educación compensatoria. Esta decisión, loable sin duda en beneficio de colectivos próximos a abandonar el sistema educativo, deslegitimó el valor de esta titulación en el imaginario colectivo.

Desde mediados de los años ochenta ha ido aumentando en España el consenso sobre la importancia de la FP y la necesidad de mejorar su calidad (UCEV, 2015). Este consenso se asienta sobre un principio diferente: la formación en empresas. Solo en 1984, cuando los primeros estudiantes de FP, antes de finalizar su formación, empezaron a adquirir experiencia laboral en empresas reales, la FP comenzó a adquirir prestigio, empezó a apreciarse la formación y la calidad del trabajo del estudiantado.

Hacia finales de los años ochenta, el Ministerio de Educación promovió comités sectoriales, a los que fueron invitados las patronales y los sindicatos, con la intención de repasar y adaptar todos los planes de estudios de FP a las necesidades del mercado de trabajo. El intento de dotar a los agentes sociales de control sobre el plan de estudios produjo buenos resultados: las 22 ramas/cualificaciones profesionales existentes se convirtieron en más de 130 títulos, con una diferenciación por niveles de cualificación intermedios y superiores, y un plan de estudios diseñado a partir de la selección de las competencias profesionales más relevantes.

Los esfuerzos realizados supusieron posteriores acuerdos: (1) en 1993, nació el primer acuerdo de la FP continua, impulsado por los sindicatos mayoritarios y el empresariado; (2) ese mismo año, se aprobó el primer Plan Nacional de Educación y Formación Profesional, renovado posteriormente en 1996; y (3) en 2002, se aprobó por consenso la Ley sobre las Cualificaciones y la Formación Profesional.

Aún hoy perduran rastros del mito sobre «los males» de la FP en España. Sin embargo, estos estigmas palidecieron ante la evidencia: la FP cualifica. Un buen ejemplo es su calidad, que ha estado fuera de dudas durante las dos últimas décadas.

Sin embargo, el acuerdo general sobre la FP se modificó unilateralmente a principios de 2012. Dos medidas impuestas por el partido conservador que gobernaba destruyeron una labor desarrollada por todos los agentes participantes en la toma de decisiones sobre esta formación. La primera medida fue la incorporación de la FP básica a la educación obligatoria con la Ley Orgánica para la Mejora de la Calidad Educativa (LOMCE, 2013), en lugar de esperar a su finalización (Marhuenda, Salvà, Navas y Abiétar, 2015). La segunda medida fue la 
aprobación hacia finales de 2012 de una normativa que establecía las condiciones de los contratos de aprendizaje e introducía la FP dual en el sistema (Real Decreto 1529/2012), cuyo objetivo era sustituir la oferta de FP por una oferta de FP dual en 2018 (Gil y López, 2013). Ni la FP básica ni la FP dual habían sido exigidas por patronales, sindicatos, el profesorado o la población joven: formaba parte de la agenda conservadora del partido gobernante, compuesta por políticas neoconservadoras y neoliberales (Abiétar, Navas y Marhuenda, 2015).

En este artículo se atenderá al desarrollo de la FP dual y se intentará mostrar que esta formación no ha logrado todos sus objetivos, al menos no en el grado que lo hizo el modelo anterior de FP. El análisis de esta implementación ya se ha documentado (Chisvert-Tarazona, Palomares-Montero y Marhuenda, 2013; Cullerés, Lletjós y Porta, 2013; Del Castillo, 2013; Gil y López, 2013), aunque se trate de un asunto en la agenda de la reforma pendiente de desarrollo.

Gran parte de la literatura producida estos años sobre la FP dual ha consistido en declaraciones y manifiestos acerca de si merecía la pena y su valor, y su utilidad y adecuación para mejorar la empleabilidad de la gente joven. El discurso oficial liderado por el Gobierno nacional y algunos gobiernos regionales, pero también por los informes realizados por instituciones como Bertelsmann (Euler, 2013) y otras (Cámara de Comercio de España, 2015a y 2015b; Frías, 2015; e IESE, 2014), traslada a la FP dual la solución a las elevadas tasas de desempleo juvenil en España.

Este artículo incluirá en su segundo epígrafe información relevante para situar el objeto de estudio de la investigación que se presenta. En el tercer epígrafe se introducirá la fundamentación teórica que permite contextualizar el estudio y mostrar los avances en este campo. En las siguientes páginas se describirá la estrategia de investigación y la metodología seguidas. Posteriormente, se presentarán los resultados preliminares de la investigación, se analizará lo que se ha descubierto y se expondrán algunas conclusiones de interés.

\section{Objeto de estudio}

Esta investigación se llevó a cabo dentro de un proyecto del programa Leonardo, en la modalidad Transfer of Innovation, cuyo objetivo es introducir la FP dual como forma de contrarrestar el abandono escolar (www.sodapprent.eu).

El hecho de que las administraciones educativas no hayan llevado a cabo hasta la fecha ninguna evaluación piloto sobre la implementación de la FP dual y que no se encuentre disponible ningún dato relevante en casi ninguna región, más allá del volumen de alumnado que cursa la formación o datos sobre su inserción propició el desarrollo de esta investigación. De hecho, los resultados proporcionados 
por el Ministerio de Educación (SGOFP, 2015) y por la Secretaría de Estado (Gomendio, 2015) eran escasos y contradictorios.

Las cuestiones que subyacen a esta investigación, por tanto, han sido las siguientes: ¿Contribuye la FP dual en la mejora de la calidad de la FP y de los centros de FP? ¿Sería más adecuada en España la FP dual que el sistema de formación profesional ordinario? La hipótesis es que la puesta en marcha de la FP dual por parte de las administraciones educativas nacionales y regionales no ha sido demandada por los centros educativos y su generalización será difícil.

\section{Fundamentación teórica‡ contexto de la reforma}

No hay mejor formación profesional que aquella arraigada con fuerza en la práctica, aquella que no pierde de vista el mundo del trabajo. Esta creencia fue la clave de la reforma educativa de 1990 Dasí como en las otras tres reformas educativas llevadas a cabo desde entonces, en 2002, 2006 e, incluso, en 2013 , que favoreció la actualización y renovación de la educación y la FP en todo el país, y se reguló en la Ley de las Cualificaciones Profesionales y la Formación Profesional de 2002.

Este cambio educativo repercute, sin duda, en el modelo curricular y organizativo de las instituciones de FP reglada. Se trata de realizar un diseño curricular desde la formación en alternancia que responda a un contexto cambiante, impredecible y temporal de los acontecimientos, para lo cual se hace necesario el cambio organizativo de la institución a través de la apertura a nuevos espacios, el establecimiento de relaciones con otros entornos y la ruptura del sincronismo espaciotemporal establecido exclusivamente dentro del aula (Huerta-Amezola, Pérez-García y Castellanos-Castellanos, 2000; Tejada, 2006).

La realización de un módulo obligatorio de experiencia en el trabajo, llevado a cabo en empresas, formaba parte de todos los planes de estudios de FP en sus diferentes cualificaciones y niveles. Además, este módulo comprendía entre el $20 \%$ y el $30 \%$ de todo el plan de estudios de cada cualificación. Por tanto, uno de los principios de la FP dual ya se había introducido con la formación en centros de trabajo (en adelante, FCT).

Parece estarse bastante de acuerdo, como ha mostrado Saniter (2015), en que la FP dual tiende a ser útil en países como Alemania, Austria, Suiza o Dinamarca, es decir, donde la economía se encuentra en desarrollo, en condiciones de estabilidad y crecimiento. La FP dual tiene más dificultades para llevarse a término cuando no se produce crecimiento económico, mientras que los sistemas de formación profesional con una participación de las empresas más limitada en los procesos de aprendizaje parecen responder mejor frente a crisis económicas. En 
2012, España todavía sufría la crisis financiera iniciada en 2007, y aún continúa vigente con elevados niveles de desempleo, especialmente juvenil.

Diferentes agentes (Unión Europea, sindicatos y corporaciones internacionales) afirman que el aprendizaje y la formación práctica en empresas son factores clave en la FP para combatir el paro juvenil. Resulta sorprendente que estas voces no atiendan al hecho de que el sistema de formación profesional español ordinario incluye la formación práctica en empresas como parte obligatoria de la formación de todos los estudiantes desde 1993. Sostienen que la FP dual producirá un cambio al ampliar la contratación del estudiantado. $\mathrm{Al}$ respecto, pueden encontrarse ejemplos en la Alianza Europea para Promover el Aprendizaje, apoyada por la Confederación Europea de Sindicatos (2014). Instituciones como la Unión Europea (2013), Bertelsmann (Euler, 2013) o las cámaras de comercio (González, 2015) también han apoyado la idea de promover la formación práctica en empresas al considerar que la mejor y única opción es, como en los países de habla germana, la FP dual. Quizá los esfuerzos realizados por el BundesInstitut für Berufsbildung (el GOVET-GRIFO) también han desempeñado un papel en este asunto. Esta institución, financiada por las cámaras de comercio el Gobierno alemán, envió delegaciones para exportar los valores de la FP dual a países tan diferentes como China, Canadá, México, Costa Rica (Gessler, 2015; Valiente, 2014 y 2015; Gonon, 2015a) o España. Esta práctica se ha criticado por las enormes dificultades que supone la transferencia de sistemas educativos a otros contextos (Deissinger, 2015; Gonon, 2015b). Una modalidad particular de estos esfuerzos, como ha mostrado Gil y López (2013), es la oferta de una FP dual alemana para jóvenes españoles -incluso si ya tienen una cualificación de FP o una licenciatura universitaria - a fin de ocupar los puestos vacantes de aprendizaje en prácticas alemanas. De este modo, se contribuye a la reducción del paro entre la juventud española.

El deseo de mejorar la educación profesional como medio para reducir el paro juvenil a través de la introducción de la FP dual es uno de los principales motivos del decreto que regula los contratos de aprendizaje y la FP dual, que se aprobó a finales de 2012. Sin embargo, el Gobierno y las administraciones de empleo no han realizado ningún esfuerzo por llevar a cabo un seguimiento apropiado de la repercusión de esta medida sobre el número de contratos de jóvenes. A pesar de los esfuerzos realizados desde esta investigación, no se ha encontrado información adecuada accesible, ni primaria ni secundaria, acerca de cómo el sistema productivo habría aprovechado los contratos de aprendizaje. Este es un descubrimiento asombroso, dado que la puesta en marcha de la FP dual en España comenzó justo cuando la crisis financiera golpeaba con más fuerza el empleo. 
Mientras los empleos desaparecían, la FP dual surgió como la tierra prometida para el paro juvenil. Sin embargo, no se llevó a cabo ninguna evaluación sobre cuántos empleos se rescataron o generaron gracias a contratos de aprendizaje ni en qué regiones o áreas profesionales.

\subsection{Las regulaciones normativas en la FP dual}

La regulación normativa de la FP y, por extensión, de la FP dual intenta repercutir sobre tres áreas. La principal es, sin duda, la mejora de la empleabilidad de los jóvenes. La siguiente en orden de relevancia consiste en la mejora de la calidad de la FP. Y, por último, de menor importancia, se encuentra la mejora del mercado de trabajo. La FP dual se plantea, por tanto, más como una política sobre el individuo - centrándose en la empleabilidad - que como una política sobre las instituciones o el contexto. La empleabilidad se considera, tal y como ocurre en la mayor parte de otros discursos (Llinares, Córdoba y Zacarés, 2011), como un rasgo personal. Esto es una negligencia respecto a la dimensión relacional de la empleabilidad, lo que contribuye a la confusión sobre el papel de la cualificación y su valor dentro del mercado de trabajo, y desplaza toda la responsabilidad sobre el eslabón más débil de la cadena: el estudiantado.

No es extraño, por tanto, que la FP dual se entienda como un medio de enriquecer la FP ordinaria, y que, al mismo tiempo, la FP dual se encuentre prácticamente ausente en la programación de la FP básica — con las únicas excepciones de Aragón, el País Vasco y Navarra-. No sorprende que la FP dual pretenda aumentar la empleabilidad de las personas que son, en principio, más susceptibles de encontrar empleo.

Hay seis regiones cuya normativa prevé una compensación económica para las empresas que participen en el sistema de FP dual: Valencia, Castilla y León, Extremadura, Galicia, Murcia y el País Vasco; aunque solo cuatro de ellas cuentan con presupuesto para ello. Asturias es la única región donde claramente se prohíbe que se pague a las empresas por su participación en la FP dual.

Cuando se plantea cómo organizar la FP dual, la mayor parte de las regiones aceptan la posibilidad de proporcionar FP dual sin firmar un contrato con el estudiantado. Tres regiones exigen en su normativa autonómica un contrato como medida necesaria para participar en la FP dual: Aragón, Castilla y León y Baleares. La mayor parte del resto de regiones aceptan una beca en lugar de un contrato o, incluso, validan que el estudiante no reciba ningún tipo de pago.

En la mayor parte de las regiones, se selecciona al estudiantado para participar en la FP dual según sus buenas calificaciones, de un modo similar a lo que solía ocurrir en el sistema de alternancia voluntario de FP a mediados de los años ochenta. 
En todas las regiones, la única responsabilidad del profesorado de los centros educativos es la evaluación. Excepto en Aragón, el personal instructor en la empresa tiene que escribir un informe sobre las tareas realizadas por el estudiante, que el profesorado puede tener en cuenta. El estudiantado de FP dual puede no realizar el módulo de FCT en Galicia, Aragón y Canarias, mientras que en el País Vasco y Valencia existe un contrato de más de un año de duración, así como en Castilla-La Mancha, donde la FCT se valida dentro del sistema de FP dual.

\section{Método}

El primer acercamiento a las fuentes oficiales de información, a los ministerios de Educación y Empleo, resultó inútil. Su información o era inaccesible o era inexistente. La información disponible no se correspondía con los datos proporcionados por las administraciones educativas. El único informe del Ministerio de Educación (SGOFP, 2015) era opaco, en el sentido de que no aportaba información valiosa sobre el proceso.

Frente a esta situación, se invirtió tiempo en la producción de una base de datos propia. Se averiguó qué centros educativos de FP estaban autorizados en cada provincia para la impartición de la FP dual, un total de 456 centros a lo largo del país. Se contactó con estos centros educativos y se comprobó si participaban en algún programa de FP dual o no. En el plazo de seis semanas se recopilaron los datos relevantes de estos centros. Mientras tanto, también se elaboraron dos cuestionarios que tenían en cuenta el tipo de agente para recoger información sobre cómo el profesorado y la dirección de los centros educativos valoraban el proceso de implementación de la FP dual.

Se envió el cuestionario por correo electrónico a todos los centros educativos en los que se impartía FP dual y se telefoneó a todos ellos para contactar con el personal directivo del centro educativo y con el profesorado responsable de la FP dual. Como resultado de este proceso, se obtuvo respuesta de las 17 comunidades autónomas, 35 provincias y 179 centros de formación, lo que incluía a 136 directores y 149 docentes responsables. Toda esta información se recopiló entre mediados de abril y mediados de junio de 2015. 


\subsection{La muestra}

Aunque el Ministerio de Educación (SGOFP, 2015) indicaba que existían 375 centros dentro del sistema de FP dual, el equipo de investigación identificó un total de 456 que se encontraban autorizados para desarrollar este sistema. De acuerdo con la participación del personal directivo, se obtuvo respuesta de al menos un tercio del total de la muestra. Las figuras por región corresponden, aproximadamente, con la implementación del sistema de FP dual en ellas, con la única excepción relevante de Madrid, donde la respuesta fue demasiado baja, y a diferencia de Castilla-La Mancha, donde hay una sobrerrepresentación en la muestra. Pueden verse las respuestas por región en la figura 1.

Figura 1. Personal directivo de centros por región.

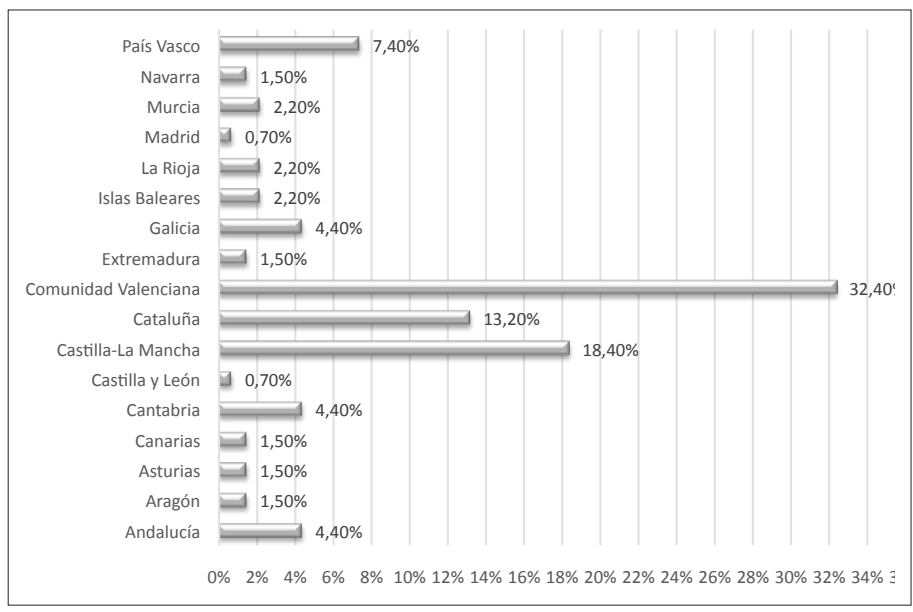

Fuente: Elaboración propia.

Se consiguieron respuestas de 149 docentes responsables de FP, aproximadamente un tercio de ellos de la Comunidad Valenciana, y de nuevo, de Cataluña y Castilla-La Mancha como regiones bien representadas, con alrededor del $15 \%$ de la muestra total cada una de ellas. Los hombres proporcionaron casi dos terceras partes de las respuestas y las mujeres, un poco más de un tercio. Más de dos terceras partes del profesorado de la muestra tenían una edad comprendida entre los 36 y los 55 años, lo que hace que el promedio de la muestra sea de 45 años. Casi dos terceras partes del profesorado poseían una licenciatura universitaria, mientras que un cuarto tenía un grado de FP.

Más de dos terceras partes del profesorado de FP dual estaban ya trabajando en el sistema de FCT, un módulo obligatorio en todos los grados de FP desde 1990, que todo el alumnado tiene que realizar dentro de una empresa, sujeto a 
evaluación y calificaciones, y para el cual es necesario haber superado otros temas obligatorios, lo que supone casi un tercio del tiempo del plan de estudios. Todo esto implica que la mayor parte del profesorado de la muestra conoce bien no solo la FP dual, sino también la FCT y, por tanto, las relaciones con las empresas al planificar la colocación, asignar el contenido y asesorar al profesorado-tutor.

Las familias profesionales con una representación más amplia en la muestra son las siguientes: dirección y administración, mantenimiento, ICT, electricidad y electrónica, comercio y marketing, así como transporte y reparación de vehículos (figura 2).

Figura 2. Principales familias ocupacionales.

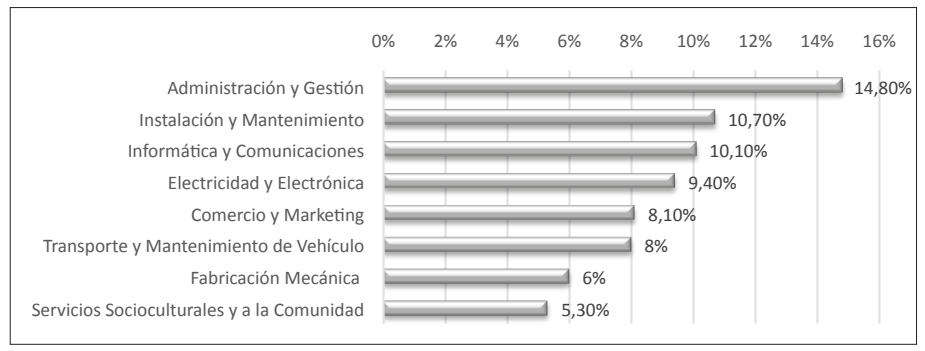

Fuente: Elaboración propia.

Una primera conclusión sobre estos datos es que la implementación de la FP dual no guarda correspondencia con las ocupaciones más demandadas. De hecho, las empresas no solo no ofrecen empleos en estas áreas, sino tampoco contratos de aprendizaje para jóvenes bajo el sistema de FP dual, dado que en muchos casos las empresas los consideran una oportunidad para reducir costes en personal. No se da una relación de enseñanza-aprendizaje y el aumento de la empleabilidad que el decreto proclama como el mayor de sus beneficios tampoco es real.

\section{Resultados y análisis}

En el siguiente apartado se aportarán algunos de los resultados más relevantes del estudio. En primer lugar, se ofrecerá una comparativa entre la FP dual y la FCT con objeto de discernir qué mejoras representa la primera. En segundo lugar, se incorporará un subepígrafe cuyo objetivo será establecer un espacio de discusión que permita poner en relación los resultados obtenidos con otras investigaciones. 


\section{1 ¿Cuál es el valor añadido de la FP dual frente a la FCT?}

La FP dual proporciona un periodo más amplio de experiencia de trabajo en las empresas. La experiencia de trabajo en empresas se reguló en España en 1974 y se aplicó por primera vez en 1983-1984 como un módulo voluntario - entonces llamado formación en alternancia-, que se transformó en obligatorio para todos los estudiantes de FP en 1990 - cuando recibió el nombre de FCT—. Para beneficiarse de la FCT, los estudiantes tenían que haber superado previamente otras materias, por lo cual la alternancia ya no era posible. Quizá la FP dual podría haber acentuado la importancia de la alternancia. Sin embargo, en la mayoría de los casos los profesores indican que la FP dual es un añadido a la FCT al ampliar las horas dentro de la empresa. Considerando este hecho, vale la pena examinar cómo la FCT y la FP dual se diferencian o complementan, analizar cuál es el valor añadido del sistema dual.

El 50,4 \% de la muestra declara que la FP dual contribuye en mucho o en gran medida a aumentar el número de estudiantes que logran la cualificación de FP, mientras que un $63 \%$ sostiene que es la FCT es la que más contribuye a lograr la cualificación de FP (figura 3). Desde este punto de vista, la FP dual no supone un valor añadido a la FCT.

Figura 3. Valoración del profesorado de FP dual relativa a la contribución de la FCT y la FP dual en la obtención del título.

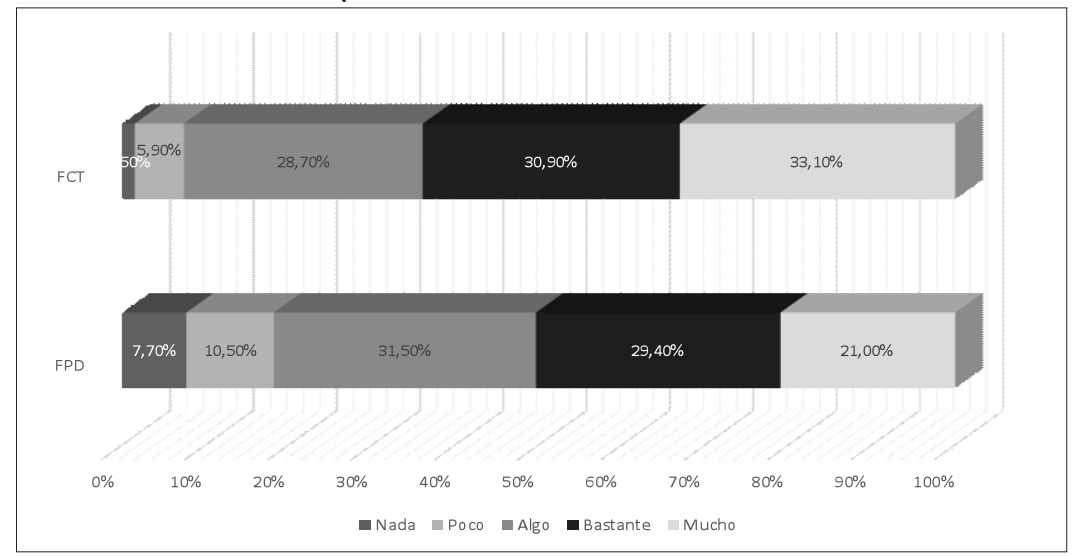

Fuente: Elaboración propia.

No se han encontrado diferencias importantes entre la FP dual y la FCT en cuanto a la motivación y la prevención del abandono de la formación por parte de los estudiantes. Aunque los resultados de ambas son similares, alrededor de dos terceras partes de las personas encuestadas consideran que la experiencia de 
trabajo en general es una buena motivación, sin importar desde qué sistema de formación se realice. Hay una leve mejora, sin embargo, cuando se trata cómo la FP dual facilita la entrada al mercado de trabajo, así como el conocimiento de la empresa (el 81,4 \% en el caso de la FP dual frente al 71,5 \% en el caso de la FCT) (figura 4).

Figura 4. Valoración del profesorado de FP dual relativa a la contribución de FP dual y de la FCT en la inserción laboral.

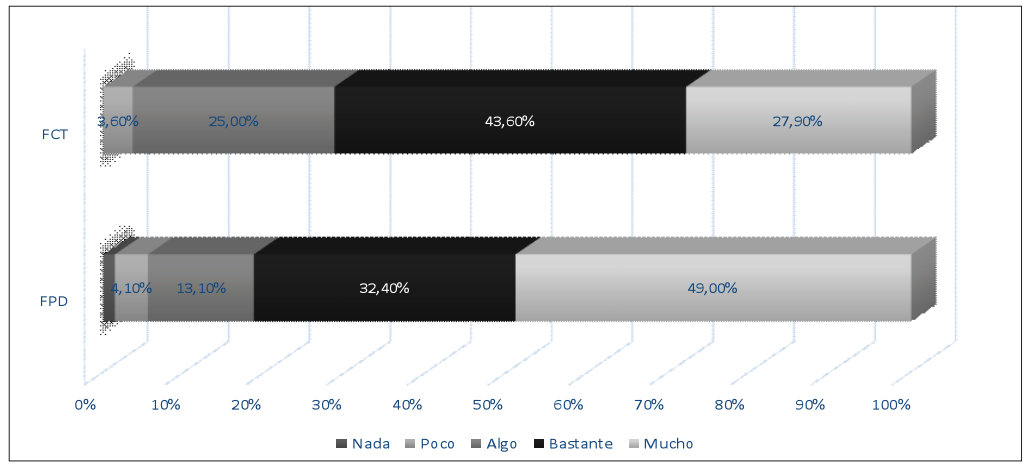

Fuente: Elaboración propia.

Asimismo, se da una mayor contribución de la FP dual al conocimiento que el profesorado tiene de la empresa específica — no del sistema productivo ni del mundo del trabajo-, así como de la responsabilidad que la empresa tiene que asumir. Tales diferencias desaparecen cuando el profesorado responde que la FP dual no mejora los procesos de planificación, dirección y desarrollo de la FP.

No se encontraron diferencias significativas entre una y otra al considerar la contribución de la FP dual en la toma de decisiones para la mejora de la FP: el conocimiento y la experiencia proporcionados por la FCT eran lo suficientemente buenos para actualizar la FP adaptando sus contenidos a las demandas de los sectores productivos.

Este resultado se reproduce en el valor académico del aprendizaje adquirido por la experiencia de trabajo, considerado por el profesorado igualmente relevante tanto en la FP dual como en la FCT y cuya diferencia es poco significativa: el $78,4 \%$ frente al $73,9 \%$.

Se encontró que una de las pocas diferencias entre ambos sistemas reside en el control de la evaluación del aprendizaje: los profesores dirigen la evaluación de la FP dual de un modo más sistemático que en la FCT (el 71,4 \%, frente al $63,8 \%)$. Sin embargo, la información proporcionada sobre la evaluación parece confusa, sino contradictoria: alrededor de la mitad de la muestra contestó que 
las competencias son objeto de evaluación, lo que implica que la otra mitad no se centra en ellas. Además, si se atiende a las herramientas utilizadas para evaluar el aprendizaje, al menos dos tercios de los encuestados se basan en la memoria del lugar de trabajo, mientras que solo el 58,1 \% se basa en la observación del desempeño. Una cuarta parte de la muestra solo realiza una evaluación final, es decir, no se comprueba el aprendizaje real de las competencias. Apenas se da una evaluación de las actitudes $(24,3 \%)$, una autoevaluación $(14,3 \%)$ o un portafolio $(7,4 \%)$, que aparecen como elementos relevantes en términos de evaluación del aprendizaje en entornos de trabajo reales.

¿Hasta qué punto la aplicación de la FP dual debe introducir cambios en las actuales prácticas de experiencia en el trabajo? El aprendizaje en competencias laborales debe preponderar sobre el valor del contenido orientador del aprendizaje, como es usual en el caso de los centros educativos. No obstante, no hacer hincapié en una evaluación sumativa es un problema. Tal vez la evaluación deba adaptarse a los perfiles ocupacionales y considerar explícitamente las competencias en las que la empresa forma al estudiantado. Posteriormente se verá si esto implica diferentes criterios de evaluación para el alumnado que prepara la misma cualificación de FP. Otra cuestión pendiente es la reticencia de las empresas a evaluar el aprendizaje del estudiante, incluso su capacidad de llevarla a cabo, en términos tanto de organización como del modo en el que la persona instructora se selecciona.

En la figura 5, se observa cómo la valoración del profesorado de FP dual encuestado en relación con las diferencias entre la FP dual y la FCT sobre sus elementos didácticos es importante. Más del 50 \% del profesorado de FP dual considera que hay bastantes o muchas diferencias en la evaluación, la metodología y los contenidos del programa formativo (figura 5).

Figura 5. Valoración del profesorado de FP dual sobre diferencias en elementos didácticos en FCT y FPD.

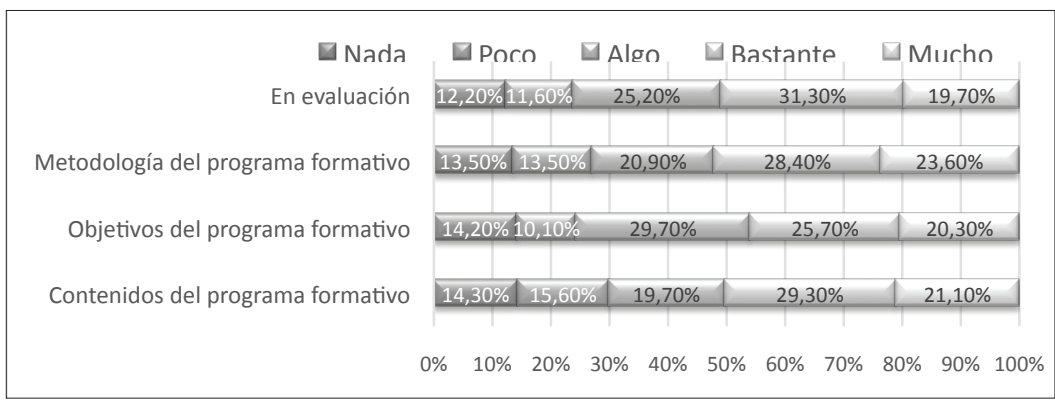

Fuente: Elaboración propia. 
La calidad del control en el lugar de trabajo debe ser un factor en la implementación de la FP dual, y se observa que al menos dos terceras partes del profesorado la consideran apropiada, de forma muy cercana a la FCT. Sin embargo, el 15,5\% del profesorado destaca la falta de control en la FP dual, ligeramente por encima de los valores correspondientes a la FCT. Resulta sorprendente el hecho de que parte del profesorado valore que no existe un intento claro de introducir indicadores para controlar y mejorar la calidad de una medida tan nueva y compleja como la FP dual.

Si se presta atención a la participación de las empresas en la FP dual, merece la pena destacar que a pesar de que el área profesional de administración es la más amplia de la FP dual, son las empresas privadas, más que la Administración pública, las que participan en la FP dual. Además, hay sectores como el de la salud y el de la educación donde, a pesar de que la Administración pública es la principal empleadora, las dificultades expuestas por las administraciones públicas para implicarse en la FP dual son considerables.

Debe advertirse la calidad de la conexión en la asignación de la FP dual en los tres diferentes niveles de cualificación de la oferta de FP. Como podría esperarse, más de cuatro quintas partes del profesorado considera la FP dual apropiada para la FP superior, aunque lo sorprendente es que el porcentaje es aún más alto para la FCT. Estos resultados son similares a los obtenidos por la FP de grado medio, aunque en este caso, la distancia entre la FP dual y la FCT es más grande y significativamente se decanta a favor de la FCT (el 85,9 \% frente al 67,7 \%). Estas semejanzas se vienen abajo cuando se trata de la FP básica, donde, de nuevo, la FCT parece más apropiada que la FP dual.

Esta tendencia se invierte a favor de la FP dual en cuanto a las oportunidades que representa para proporcionarles a los estudiantes el conocimiento técnico requerido en el mundo del trabajo. No obstante, aunque las diferencias no pueden considerarse significativas, existen. Las diferencias se tornan relevantes al comparar cómo la aplicación de la FP dual favorece el contacto con el mundo de trabajo en una medida mucho mayor que la FCT (el 72,2 \% frente al 55,4 \%).

Para concluir con esta presentación de resultados tras la comparación de la FP dual con la FCT, los datos confirman que la primera está mejor valorada por el profesorado que la lleva a cabo porque favorece las relaciones y el conocimiento mutuo entre instructores, trabajadores y aprendices. Más de la mitad del profesorado lo señala, frente al $40 \%$ que se inclina a favor de la FCT. Estos resultados y diferencias no son tan claros en cuanto a las relaciones entre empleadores y aprendices. Cabe destacar que casi la mitad del profesorado declara que la FP dual aumenta la capacidad de adaptarse a las demandas del mercado de trabajo, mientras que solo el 28,6 \% mantiene esta afirmación para la FCT. 


\subsection{Discusión y sugerencias para investigaciones posteriores}

Los motivos para llevar a cabo la implementación de la FP dual en España son diversos y variados en su alcance, tanto como los agentes implicados (la administración educativa, empresas, agencias de control simbólico como fundaciones internacionales, cámaras de comercio, el profesorado y el propio estudiantado). La presión y la precipitación de la Administración nacional y de algunas administraciones regionales por adaptarse a la reforma propuesta han causado muchos problemas a lo largo del proceso de adaptación. Parece que el sistema se ha agotado en una tentativa que ha desviado esfuerzos de otras áreas de interés y necesidades para mejorar la FP. El proceso de implementación ha sido irregular y no se ha normalizado, lo que ha provocado confusión también entre las empresas invitadas a participar en la FP dual. La desregulación del mercado de trabajo - más de 36 reformas en los últimos 40 año- parece encontrarse ahora acompañada por la desregulación del sistema de educación profesional, al que han contribuido tanto la FP básica como la FP dual.

Desde luego, nadie se atrevería a oponerse a la idea de un sistema dual, la noción misma es una marca, como algunos han mostrado (Heikkinnen y Lassnigg, 2015; Lassnigg, 2015; Marhuenda, 2015), y es una buena marca, con valor en sí misma. La FP dual en su tradición centroeuropea parece funcionar correctamente y estar bien consolidada, :por qué no debería funcionar aquí igual de bien? No es solo desde el ámbito político y técnico desde donde se defiende este discurso, también el profesorado considera que puede ser beneficioso para ciertos estudiantes. No puede olvidarse que la FP ha sido una de las mayores áreas de innovación pedagógica en los últimos 20 años en España y, por tanto, el profesorado se encuentra preparado para formar parte de la experimentación y para modificar sus prácticas tradicionales, como ya han hecho frecuentemente en el pasado. Mientras que en el resto de niveles del sistema educativo, las reformas se han centrado principalmente en el currículo, en el caso de la FP, la organización de los servicios educativos ha sido objeto de cambios, en una constante búsqueda de mejora e innovación.

Así pues, la cuestión no es si la FP dual debe rechazarse - que no es el caso, sino si intenta complementar el modelo de FP existente, o bien sustituirlo y reemplazarlo por algo diferente y ajeno a la cultura de la FP española. Tendría sentido, por tanto, centrarse en las condiciones que hacen posible la implementación de la FP dual como un complemento al sistema de formación profesional que es - no puede olvidarse - de base escolar, pero con la particularidad de que la experiencia laboral está firmemente arraigada como módulo obligatorio en las empresas. Por ello, deben mencionarse tanto a las empresas como al estudiantado, y 
quizá habría que buscar las diferencias existentes entre los sectores profesionales. La segunda opción, el reemplazo del actual sistema de formación por uno dual, incluso aunque el Ministerio de Educación lo haya anunciado en sus proyectos políticos, no parece posible: los principales partidos políticos y agentes sociales han negociado y acordado durante mucho tiempo el sistema de formación profesional español, no existe ninguna demanda de cambio y, por tanto, no hay un apoyo suficiente para una generalización de la FP dual.

Otra pregunta que debe responderse es si el modelo de aprendizaje puede variar sin que se introduzcan cambios en los fundamentos pedagógicos que lo sostienen. Desde luego, esto no será posible si no se tiene en cuenta una mayor participación de las empresas, lo que no se ha considerado hasta ahora en el proceso de implementación de la FP dual. Quizá esta cuestión se relaciona con la escasa cultura de aprendizaje y educación, que no se ha integrado lo suficiente en el espíritu emprendedor que se promueve en el país.

En esta investigación se ha observado que se ha alterado el plan de estudios, se ha deformado y reorganizado para la totalidad de estudiantes de FP a favor de un pequeño grupo: el de los escasos beneficiaros del sistema dual.

\section{Conclusiones}

Se ha intentado mostrar los resultados siempre desde la perspectiva del profesorado implicado en la FP dual y teniendo en cuenta las regulaciones relacionadas con la FP de las diferentes regiones. Ahora se puede retomar la pregunta inicial: ¿la FP dual es algo más que horas de experiencia de trabajo, que una ampliación de la FCT? El carácter obligatorio de la FCT le permitió llegar a la totalidad de los estudiantes. Por tanto, ¿necesita el sistema de formación profesional español un sistema dual?

Quizá esta pregunta llega tarde, ya que este sistema se introdujo hace más de tres años y ha sido ampliamente anunciado - en realidad, más anunciado que puesto en marcha-. La pregunta, entonces, no es tanto si puede o debe eliminarse la FP dual, sino más bien cómo hacerla valiosa como contribución adicional a la oferta voluntaria de la FP para estudiantes.

A pesar de que no existía una clara demanda por parte de las empresas, lo que ha conducido a una falta de participación apropiada, y a pesar de que la Administración ha empujado al profesorado a la FP dual sin proporcionarle el apoyo adecuado, no se tiene la intención de forzar la abolición de la FP dual. Sin embargo, existen, desde luego, algunas lecciones que pueden aprenderse sobre cómo este proceso de implementación ha incurrido en errores que deberían evitarse en 
cualquier futura reforma de la educación profesional en el país e, incluso, podrían dar pie a mejoras en el sistema en proceso de implementación.

Primero, es clave reunir a todos los agentes sociales y acordar con ellos las principales necesidades y las áreas de mejora de la FP, en lugar de promover una reforma que se decide desde las altas instancias sin contar con los agentes implicados y, por tanto, sin garantizar el acuerdo de aquellos que tienen que acogerla.

Segundo, debe dedicarse tiempo a introducir un nuevo sistema complejo que requiere la participación real de diferentes agentes que no están acostumbrados a él y que no lo han demandado. La competición en la que algunas administraciones regionales han entrado, junto con el Ministerio de Educación, con el fin de ver quién era la primera en obtener las cifras más altas de estudiantes participantes, ha sido contraproducente en términos del prestigio de la FP dual. Se ha precipitado, se ha acelerado, su puesta en marcha y se ha olvidado la planificación y el seguimiento de medidas de control. Todo esto ha reducido la calidad del proceso y ha causado confusión y presión entre el estudiantado de FP, las empresas, los agentes sociales y las familias, así como desigualdades entre el alumnado dentro del mismo sistema de formación profesional.

Ha sido particularmente preocupante actuar en nombre de las empresas sin tenerlas en cuenta al enviarles profesorado en calidad de comerciales para venderles una idea que debería haber nacido de las propias empresas y no de la Administración. Las administraciones no escucharon las demandas de las empresas, sus sugerencias o requerimientos, y el sistema se puso en marcha sin su acuerdo; un sistema flexible, desde luego, pero muy confuso, debido a la falta de definición y a la enorme desregularización en la legislación estatal. La FP dual sin la iniciativa privada, sin contar con la empresa, es incapaz de universalizarse.

La FP dual aterrizó en el sistema de formación profesional como algo no demandado, inesperado, pero fue rápidamente aceptada. Nadie la rechazó y tanto los agentes sociales como el profesorado trataron de hacerlo lo mejor posible con ella. El proceso de implementación se desarrolló en medio de una mezcla de miedo, imposición, innovación y buenas intenciones, especialmente teniendo en cuenta que las personas jóvenes podrían beneficiarse de ella en un momento de altos niveles de desempleo juvenil.

El resultado hasta ahora es claro: se espera de una escuela basada en un sistema de FP dual que los centros escolares de FP y su profesorado sean los responsables de buscar empresas, explicarles de qué trata la FP dual y convencerles de las ventajas para ellos - y para los estudiantes - reorganizando el plan de estudios y la organización de la FP, y redactando para las empresas planes curriculares individuales, así como criterios de evaluación. Los centros educativos 
y el profesorado han sido los responsables de implementar la FP dual, pero no han contado ni con los recursos ni con la autonomía para adaptarla a las circunstancias de su entorno, lo que también ha causado diferencias importantes entre los centros educativos de FP en términos de oferta y potencial atractivo para los nuevos estudiantes.

A pesar de lo irresponsable que esto pueda parecer, se ha logrado, sin embargo, abrir la puerta a la FP dual en un sistema basado en la escuela y encontrar un lugar para ello, más allá del módulo obligatorio de experiencia de trabajo, la FCT. Si esta puede ampliarse, generalizarse, muchas cosas deberían cambiarse para garantizar su calidad.

Cabe destacar también que la FP dual ha sido incapaz de generar empleo; su contribución al aumento de la empleabilidad solo puede medirse en términos individuales. Además, se introdujo en el peor momento posible: el de la repercusión más profunda de la reciente y duradera crisis financiera. Su puesta en marcha ha sido selectiva para los estudiantes, realizada voluntariamente por el profesorado, que se ha enfrentado con la desilusión y ha tratado de contribuir a las posibilidades de aprendizaje de sus estudiantes. Las administraciones han impulsado el sistema desde sus oficinas en lugar de escuchar las demandas de los agentes sociales. Estos, junto con el profesorado, han reaccionado del mejor modo posible sin ser proactivos y en un periodo de productividad limitada debido a la crisis económica. La FP dual es una buena idea que, sin embargo, ha sido mal implementada en España.

El resultado es que la $d$ de dual representa la $d$ de duda, distracción y desregularización. No ha sido demandada por ningún agente educativo o profesional ni tampoco adecuadamente dirigida, no ha sido deseada, permanece desconocida en gran medida y es débil en su funcionamiento. La FP dual se ha devaluado por el modo en el que se ha implementado. Quizá no era necesaria en un sistema de formación profesional como el español. Puede que ahora la cuestión sea cómo hacerla útil, productiva, responsable y sostenible en nuestro contexto. 


\section{Referencias}

Abiétar, M.; Navas, A.; y Marhuenda, F. (2015): «Aportaciones desde la justicia social para una educación justa. La identidad pedagógica en formación profesional básica», Revista Internacional de Educación para la Justicia Social, 4 (2), pp. 145-161.

Cámara de Comercio de España (2015a): Factores de éxito de la formación profesional dual, Madrid: Cámara de España.

- (2015b): Guía de formación dual, Madrid: Cámara de España.

Chisvert-Tarazona, M. J.; Palomares-Montero, D.; y Marhuenda, F. (2013): «La letra pequeña en los contratos de aprendizaje. Lo que no se dice», Organización y Gestión Educativa, 3, pp. 19-22.

Confederación Europea de Sindicatos (ETUC) (2014): Towards a European quality framework for apprenticeships and work-based learning, en línea. $<$ www.etuc.org/sites/www.etuc.org/files/publication/files/ces-brochure_ unionlearn-uk-rouge.pdf $>$.

Cullerés, M. Á.; Lletjós, F.; y Porta, S. (2013): «Puesta en marcha de la formación dual», Organización y Gestión Educativa, 3, pp. i-viii.

Deissinger, T. (2015): Transferring the dual system to the Canadian contextdo German employers in Ontario adopt the German model?, paper presented at the 22nd Conference for Vocational and Education and Culture-research network: Vocational education, citizenship and participation: Problematizing relations between education, work and politics from contemporary and historical perspectives, Valencia, del 4 al 6 de julio.

Del Castillo, M. (2013): «Primera visión sobre la formación dual en España», Organización y Gestión Educativa, 3, pp. 13-17.

Euler, D. (2013): El sistema dual en Alemania: ¿Es posible transferir el modelo al extranjero?, Madrid: Bertelsmann.

FríAs, J. (2015): La calidad en la formación profesional dual, Seminario de Formación Dual, Madrid: Cámara de Comercio de España.

Gessler, M. (2015): Dual abroad: application, adaptation or innovation. Apprenticeship training at the Mercedes-Benz plant in Tuscaloosa, USA. A case study, Paper presented at the IV International Conference $\&$ Research Workshop Vocational Education \& Training. Emerging Issues? Voices from Research, Estocolmo.

GiL, G. y López, A. (coords.) (2013): Formación profesional dual: algo más que un decreto, Valencia: AREA.

Gomendio, M. (2015): La formación profesional en España, Madrid: MEC. 
Gonon, P. (2015a): «Are apprenticeships exportable? The debate on transferring dual systems», paper presented at the 22nd Conference for Vocational and Education and Culture-research network: Vocational education, citizenship and participation: Problematizing relations between education, work and politics from contemporary and historical perspectives, Valencia.

- (2015b): «Exporting the dual system (apprenticeship system)? Promises and pitfalls», paper presented at the IV International Conference \& Research Workshop Vocational Education \& Training. Emergint Issues? Voices from Research, Estocolmo.

González, E. (2015): Los retos en el desarrollo de una formación profesional dual de calidad, Madrid: Cámara de España.

Heikninen, A. y Lassnigg, L. (eds.) (2015): Myths and brands in vocational education, Newcastle upon Tyne.

Huerta-Amezola, J.; Pérez-García, I. S.; y Castellanos-Castellanos, A. R. (2000): «Desarrollo curricular por competencias profesionales integrales», Revista Educar, abril-junio, pp. 87-96.

IESE (2014): La formación profesional dual como reto nacional. Una perspectiva desde la empresa, en línea. <www.iese.edu/research/pdfs/ST-0362.pdf>.

LASSNIGg, L. (2015): «The political branding of apprenticeship into the "dual system"-Reflections about exporting the myth of employment transition», en A. Heikninen y L. Lassnigg (eds.) (2015): Myths and brands in vocational education, Newcastle upon Tyne, pp. 78-99.

«Ley Orgánica 8/2013, de 9 de diciembre, para la Mejora de la Calidad Educativa», Boletín del Estado (BOE), 295, 10 de diciembre de 2013. Disponible en el siguiente enlace: <www.boe.es/diario_boe/txt.php?id=BOEA-2013-12886>.

Llinares, L.; Córdoba, A.; y Zacarés, J. J. (2011)ः «La medida de la empleabilidad desde las empresas de inserción social», en A. Córdoba e I. MartíNEZ (coords.) (2011): Trabajo, empleabilidad y vulnerabilidad social, Valencia: Universitat de València, pp. 131-182.

Marhuenda, F. (2015): «Vocational education abused: precarisation disguised as dual system», en A. Heikninen y L. Lassnigg (eds.) (2015)ः Myths and brands in vocational education, Newcastle upon Tyne, pp. 59-77.

Marhuenda, F; Salvà, F; Navas, A.; y Abiétar, M. (2015): «Twenty years of basic vocational education provision in Spain: changes and trends», International Journal for Research in Vocational Education and Training, 2 (2), pp. 137-151. 
«Real Decreto 1529/2012, de 8 de noviembre, por el que se desarrolla el contrato para la formación y el aprendizaje y se establecen las bases de la formación profesional dual», Boletín Oficial del Estado (BOE), 70, 9 de noviembre de 2012.

Saniter, A.; Tutlys, V; y Marhuenda, F. (2015): «The potential of implementation of work process based propaedeutic apprenticeship schemes in the VET systems of Germany, Spain and Lithuania», en M. Gessler y L. Freund (eds.) (2015): Crossing boundaries in vocational education and training: innovative concepts for the 21st century, Bremen: ITB, pp. 112-116.

SGOFP (2015): Formación profesional dual. Seguimiento de proyectos FPD curso 2013-2014, en línea, Madrid: MEC. <www.mecd.gob.es/prensa-mecd/ $\mathrm{dms} / \mathrm{mecd} /$ prensa-mecd/actualidad/2014/01/20140113-fpdual-bankia/ informe-seguimiento-fpdual.pdf $>$.

TEJADA, J. (2006): «Elementos de convergencia entre la formación profesional y la universidad: implicaciones para la calidad de la formación profesional superior», Revista de Educación, 340, pp. 1085-1117.

Unión de Cooperativas de Enseñanzas Valencianas (UCEV) (2015): La sociedad necesita una formación profesional para la vida, Valencia: UCEV.

Unión Europea (2013): Apprenticeship and traineeship schemes in EU27: key success factors, en línea, Luxemburgo: Unión Europea. <ec.europa.eu/education/policy/vocational-policy/doc/alliance/apprentice-trainee-successfactors_en.pdf $>$.

Valiente, Ò. (2015): «A Mexican model of dual VET? Adoption and recontextualization of a global education policy», paper presented at the 22nd Conference for Vocational and Education and Culture-research network: Vocational education, citizenship and participation: Problematizing relations between education, work and politics from contemporary and historical perspectives, Valencia.

- (coord.) (2014): Un model de formació professional dual per a Catalunya: reptes en el disseny $i$ implementació de la reforma, Barcelona: Jaume Bofill. 